\title{
Validated Electromagnetic Coupling Predictions for an Electronic Subsystem Based on the Finite-Element Time- Domain Method
}

\author{
Douglas Riley ${ }^{1}$, Gary Sower ${ }^{2}$, David Schafer ${ }^{3}$ and Gerald Baca ${ }^{3}$ \\ ${ }^{1}$ Northrop Grumman Corporation, 100 Sun Avenue NE, Suite 300 \\ Albuquerque, NM 87109 (douglas.riley@ngc.com) \\ ${ }^{2}$ ITT Industries, Advanced Engineering \& Sciences Division, \\ 5901 Indian School Road NE, Albuquerque, NM 87110 \\ ${ }^{3}$ Air Force Research Laboratory, 3550 Aberdeen Ave SE \\ Kirtland Air Force Base, NM 87117
}

\begin{abstract}
The finite-element time-domain (FETD) method is used to predict electromagnetic coupling into an electronic subsystem. Accurately predicting coupling into electronics systems is challenging because small changes to geometry and materials can significantly shift the frequencies at which resonances and nulls occur. The test object consisted of two metal chassis, a variety of apertures, an unshielded wire, multipin connectors, Eccosorb ${ }^{T M} \mathrm{RF}$ absorptive sheets, and circuit boards. Experimental validation is provided over a frequency range of $0.5 \mathrm{GHz}$ to $3 \mathrm{GHz}$. The experimental response of the test object was based on illumination by a double ridge horn antenna at distances of $1 \mathrm{~m}$ and $7.5 \mathrm{~m}$, whereas the predictions were based on free-field, plane-wave excitation. Good correlation between measurements and predictions for the amplitude and trend of the coupling data is established down to the circuit-trace level.
\end{abstract}

\section{INTRODUCTION}

It is often impractical to test full systems under free field illumination for a wide spectrum of coupling scenarios. However, isolated testing of subsystems is commonplace. Although the response of the subsystem to free-field illumination is often desired, practical limitations can restrict the testing to direct injection techniques, or possibly external antenna illumination with the antenna placed relatively close to the test object ("spot" illumination). It is of interest to understand the relationship of the data obtained from these testing approaches to the response that would be obtained under a true free-field illumination environment. Analytical and numerical experiments are becoming increasingly important for obtaining the free-field response. The purpose of this paper is to report on coupling correlation between plane-wave illumination based on transient finite-element methods and spot-illumination response measurements over a frequency range of $0.5 \mathrm{GHz}$ to $3 \mathrm{GHz}$.

For highly controlled systems at relatively low frequencies, it is currently possible to predict the electromagnetic response to a very high level of correlation with experimental measurements. For more practical applications, and/or as frequencies extend into the overmoded region, pointwise correlation between predictions and measurements becomes increasingly impractical because minor geometry and material variations can significantly shift the frequency locations of resonances and nulls. Instead, correlation of the bound and trend of the data often represents a realistic goal for internal coupling applications.

\section{ELECTRONIC SUBSYSTEM TEST OBJECT}

The test object is shown in Fig. 1. The geometry consists of two metal chassis that are interconnected by an unshielded (but insulated) 22 AWG wire. This wire enters the two chassis through multipin connectors and ultimately attaches to traces on internal circuit boards. Eccosorb $^{T M}$ RF absorptive sheets were used to significantly lower the $Q$ of the two chassis (from several hundred to approximately sixty). The chassis were placed on a $4^{\prime} \times 8^{\prime}$ aluminum ground 
plane. The "Primary Chassis" shown in Fig. 1 has two, 6-inch linear slots, and one, 1-in circular aperture. These apertures were either open or closed depending upon the test configuration.

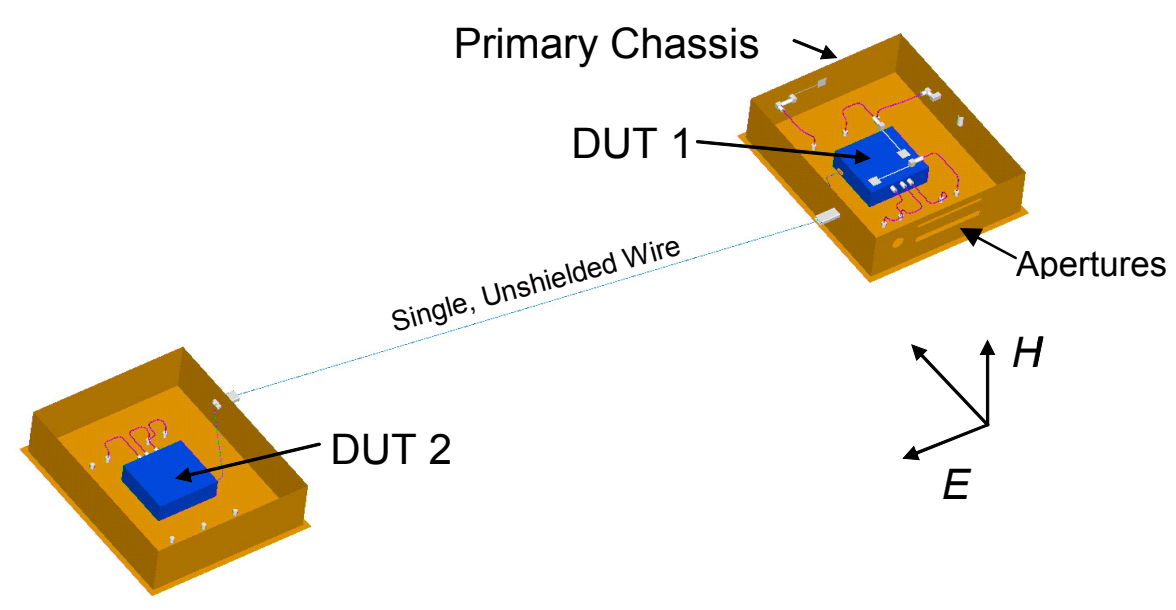

Figure 1. Cut-a-way view of test object. Test object placed on 4'x8' ground plane. Eccosorb ${ }^{T M}$ RF absorptive sheets placed on inside top surfaces of chassis.

A solid model and finite-element mesh of the test object were carefully constructed. Although not every detail of the actual test object was included in the model, the primary features were included and these are estimated to be located within $+/-0.5 \mathrm{~cm}$ of the actual test object However, the proper definition of the material parameters, particularly over wide frequency bands, is challenging. This is particularly true for the Eccosorb ${ }^{T M}$ material sheets. Often manufactures only provide reflection coefficient data, whereas for an accurate model, the precise complex permittivity and/or permeability are required. Thus, even when the geometrical features are well characterized, material uncertainties can degrade the overall fidelity of the model. Additional graphics of the internal details and internal mesh of the test object are shown in Figs. 2(a),(b).

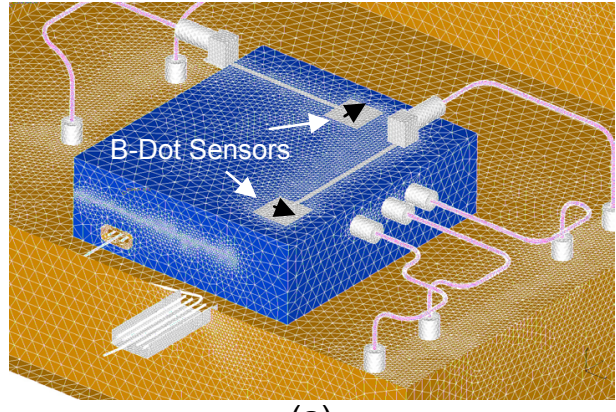

(a)

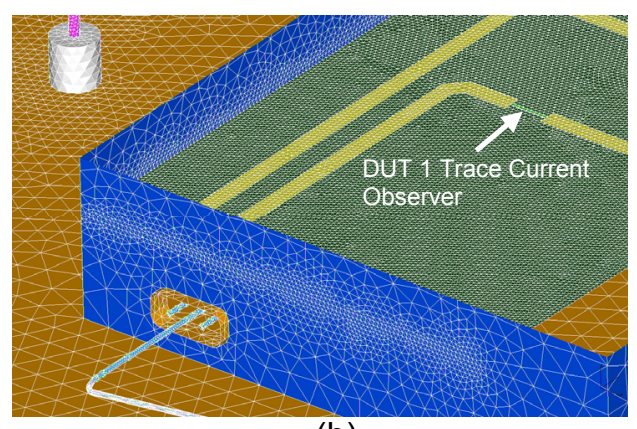

(b)

Figure 2. (a) Internal finite-element mesh. (b) Internal circuit board trace observer.

\section{FINITE-ELEMENT TIME-DOMAIN METHOD}

The wave equation in a volume $\Omega$ bounded by a closed surface $\partial \Omega$ in terms of the timedependent total electric field, $\boldsymbol{E}(\boldsymbol{r}, t)$, is given by [1]

$$
\nabla \times\left[\frac{1}{\mu} \nabla \times \boldsymbol{E}(\boldsymbol{r}, t)\right]+\varepsilon \frac{\partial^{2}}{\partial t^{2}} \boldsymbol{E}(\boldsymbol{r}, t)+\sigma \frac{\partial}{\partial t} \boldsymbol{E}(\boldsymbol{r}, t)+\frac{\partial}{\partial t} \boldsymbol{J}(\boldsymbol{r}, t)=0 \quad \boldsymbol{r} \in \Omega
$$


where $\boldsymbol{J}$ denotes an electric current density, and $\varepsilon, \mu, \sigma$ denote permittivity, permeability, and conductivity, respectively. By using vector edge basis functions for the electric field, the wave equation is written as the following semi-discrete matrix equation

$$
M \frac{d^{2}}{d t^{2}} \mathbf{E}+K \frac{d}{d t} \mathbf{E}+S \mathbf{E}+\mathbf{f}=0
$$

where $M, K$, and $S$ denote the usual finite element matrices [1], and $\mathbf{f}$ denotes a forcing function. To improve the efficiency of the solution, the FETD formulation was hybridized with the finitedifference time-domain (FDTD) method using the approach described in [2]. In addition, parallel domain-decomposition techniques were used to partition both the FETD and FDTD regions across multiple computer processors.

\section{MEASUREMENT SETUP}

The basic measurement setup is shown in Fig. 3(a). The low-level testing as a function of frequency required a network analyzer, cables, a double ridge waveguide horn radiating element, B-dot and D-dot free-field sensors to confirm the external field level, internal B-dot and D-dot surface sensors, two Tektronix CT-6 current probes for measurements on the printed circuit boards (PCBs), and a test stand that allowed a large range of azimuth angles of illumination. Voltage probes were built onto the PCBs as resistive voltage dividers. Internal field measurements were made to determine the transfer functions for the various plane wave and spot illuminations. Current and voltage measurements were made on the PCBs to determine the transfer functions to the board level. The HP8753C network analyzer was operated from $0.5 \mathrm{GHz}$ to $3 \mathrm{GHz}$. Measurements were taken at $1 \mathrm{~m}$ and $7.5 \mathrm{~m}$ from the test object. When normalized by the propagation distance, the $7.5 \mathrm{~m}$ (true far-field) data and the $1 \mathrm{~m}$ (relative near-field, or spot illumination) data typically differed by $2 \mathrm{~dB}$ to $6 \mathrm{~dB}$ over the frequency range, with the differences primarily in the depths of nulls and height of peaks (c.f. Fig. 3(b)). In the following section, the 1$\mathrm{m}$ measurement data is used for comparison with the free-field predictions, since this may be more representative of the practical situation where one is limited to a near-field measurement environment yet desires to know the relationship of these data to a free-field environment

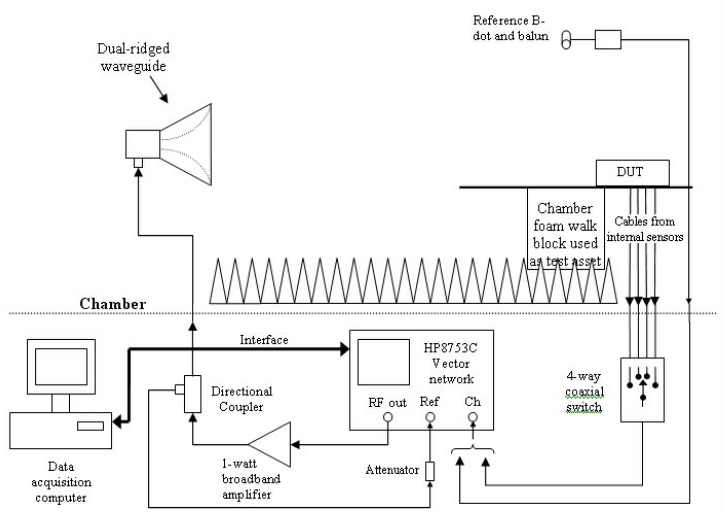

(a)

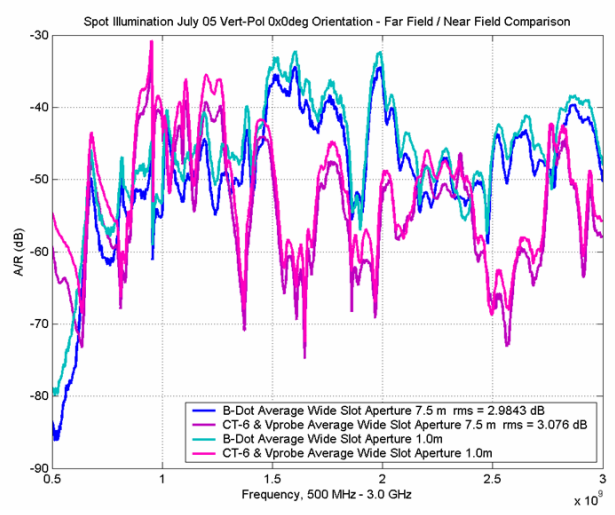

(b)

Figure 3. (a) Measurement setup. (b) Comparison of raw measured data for B-Dot and current sensors at $1 \mathrm{~m}$ and $7.5 \mathrm{~m}$ for the case that the wide slit aperture was open.

\section{RESULTS}

Several test configurations were examined. These included different choices of open apertures as well as different illumination angles. A few representative results are included in this section. Fig. 4(a) shows a transfer function that relates the coupling to the internal B-Dot sensors in the 
primary chassis (c.f. Figs. 1,2(a)) to the incident magnetic field for the case that one of the two 6in. slot apertures was open $(0.5$-in. slot width). The incident wave was vertically polarized and normally incident on the front, or aperture face, of the primary chassis. Results for coupling to the internal circuit board trace observer (c.f. Fig. 2(b)) are shown in Fig. 4(b) for the case of normal incidence and horizontal polarization. Note that the latter case is cross-polarized to the slot and the average trace-current level is on the order of $-100 \mathrm{dBA}$. Similar results for the 1-in. circular aperture are shown in Figs. 5(a),(b) for the case of normal incidence and vertical polarization. A linear fit to the data is provided in the figures.
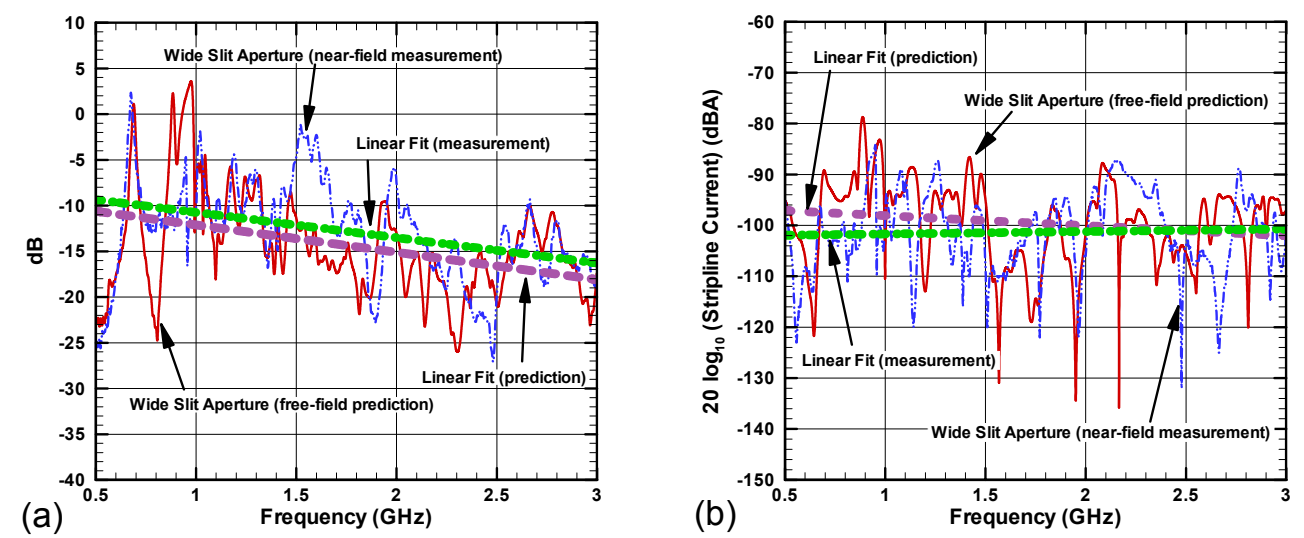

Figure 4. Open slit aperture. (a) B-Dot sensors transfer function. (b) Trace current.
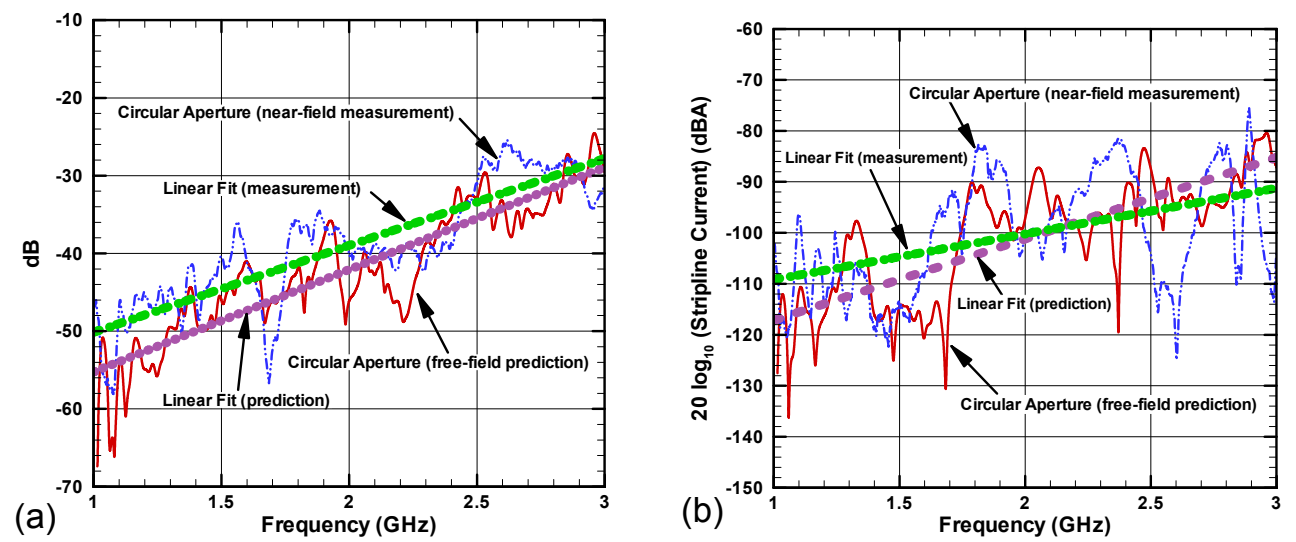

Figure 5. Open circular aperture. (a) B-Dot sensors transfer function. (b) Trace current.

\section{CONCLUDING REMARKS}

Accurately predicting coupling to interior electronics systems is challenging. Small changes to geometry and materials can significantly shift the frequencies at which resonances and nulls occur. Nevertheless, it is expected that the overall amplitude, trend, and statistics of the interior coupling data will remain relatively constant between similar systems. This has been established through the data comparison of predictions and measurements for the electronics subsystem test object, even with induced trace current levels on the order of $-100 \mathrm{dBA}$.

\section{REFERENCES}

[1] Jin, J.-M., The Finite Element Method in Electromagnetics, 2nd Ed., New York: Wiley, 2002.

[2] Rylander, T., F. Edelvik, A. Bondeson, and D. Riley, "Advances in Hybrid FDTD-FE Techniques," Computational Electromagnetics: The Finite-Difference Time-Domain Method, A. Taflove and S. C. Hagness, Editors, $3^{\text {rd }}$ Edition, Artech House, 2005, pp. 907-953. 